\title{
Cómo están pasando la pandemia los estudiantes de la Universidad Pedagógica Veracruzana: un estudio de caso
}

\author{
How students of Universidad Pedagógica Veracruzana are \\ going through the pandemic: a case study
}

DOI: https://doi.org/10.32870/dse.v0i22.816

\author{
Erick Hernández Ferrer* \\ Óscar Valencia Aguilar**
}

\begin{abstract}
Resumen
En el presente artículo se describe quiénes son los estudiantes de la Universidad Pedagógica Veracruzana y cómo están pasando la emergencia sanitaria derivada del COVID-19, con la finalidad de dar cuenta de sus estrategias, motivaciones y prácticas durante el confinamiento; para ello hemos realizado una serie de encuestas, registros de observación y entrevistas semiestructuradas dirigidas a una muestra de un total de 82 alumnos de la institución, de los niveles de licenciatura, maestría y doctorado; por lo que se desarrolló un enfoque multimétodo. Así, a partir de los datos obtenidos, realizar un análisis de política de tipo posdecisional con el propósito de señalar los logros alcanzados por la misma universidad a raíz de lo encontrado con sus estudiantes, entre los que se destaca que, si bien la institución educativa respondió de manera inmediata, esto no ha sido suficiente respecto a los contextos personales y profesionales de sus estudiantes, pues parece que solo se realizó una especie de sobreposición de los contenidos curriculares para su abordaje ya que, al no contar con una plataforma institucional activa, los docentes tuvieron que utilizar diversas herramientas tecnológicas (Zoom, Meet, Skype, edmodo, entre otros) según sus consideraciones.
\end{abstract}

Palabras clave: prácticas educativas - confinamiento - análisis de política - sujetos - institución educativa.

\begin{abstract}
This article describes who the students of the Universidad Pedagógica Veracruzana are and how they are going through the health emergency derived from COVID-19, in order to account for their strategies, motivations and practices during the lockdown. We conducted a series of surveys, observation records and semi-structured interviews with a sample of a total of 82 students from the institution at the bachelor's degree, master's and doctoral levels, using a multi-method approach. Based on the data obtained, we carried out a post-decision type policy analysis with the aim of pointing out the university's performance
\end{abstract}

\footnotetext{
* Doctor en Investigación Educativa. Universidad Pedagógica Veracruzana. México. ehferrer@gmail.com

** Doctor en Política Educativa y Evaluación. Universidad Pedagógica Veracruzana México. o.valencia.aguilar@gmail.com
} 
as a result of our findings with its students, one of which was that, although the university's response was immediate, it did not take into account the students' personal and professional contexts: apparently, there was only a sort of overlapping of the curricular contents to be addressed, since due to the lack of an active institutional platform the teachers had to resort to the use of technological tools such as Zoom, Meet, Skype, and edmodo, among others.

Keywords: educational practices - confinement - policy analysis - subjects - educational institution.

\section{Introducción}

En el marco del seminario institucional y del programa de formación en investigación social y educativa (SISE), nos hemos dado a la tarea de realizar una investigación titulada: Constitución y formación de sujetos universitarios en contextos de crisis, con la finalidad de dar a conocer las dinámicas en las que los estudiantes de la Universidad Pedagógica Veracruzana (UPV) se están formando, no solo como estudiantes sino como sujetos.

Lo anterior muestra una franca relación con la situación actual desatada por la contingencia sanitaria por la epidemia de COVID-19, por lo que la hemos tomado como eje de análisis, entendiéndola como el punto nodal de un contexto en el que se desarrollan múltiples crisis y, en consecuencia, afecta al campo de lo educativo.

Ahora bien, se revisaron estudios nacionales como el de Mérida y Acuña (2020) y casos internacionales como los expuestos por Álvarez, Gardyn, lardelevsky y Rebello (2020), Expósito y Marsollier (2020), en los que muestran elementos de análisis sobre educación básica y se describen problemas de segregación educativa que reflejan el uso de recursos pedagógicos predominantes, y advierten que el uso de diferentes tecnologías no significa que se hayan superado las desigualdades de acceso a la educación, que coinciden con los hallazgos en el presente estudio, pero no encontramos referencias a la educación superior.

Después de hacer una revisión de los documentos oficiales emitidos por la Secretaría de Educación Pública (SEP), publicados a nivel nacional para atender a la comunidad estudiantil de México, se encontró que estos solo estaban dirigidos para el nivel básico, como la estrategia "Aprende en Casa", que implementó la SEP (2020) para los niveles de educación inicial, preescolar, primaria y secundaria y, a través de la Estrategia de Educación a Distancia, estableció que niñas, niños y jóvenes continuaran "con sus aprendizajes escolares durante el periodo de contingencia sanitaria nacional a través de las herramientas que ofrecen Google y YouTube para Educación" (SEP, 2020). La cual se abocó a trasmitir clases en canales televisivos, publicar materiales educativos y hacer uso intensivo de tecnologías educativas (clases grabadas en la aplicación YouTube, aulas virtuales mediante Google Classroom, entre otros).

Diálo@os sobre Educación año 12 | número 22 | enero-junio 2021 | ISSN 2007-2171 
Otro elemento de análisis fueron las estrategias desarrolladas por la Secretaría de Educación de Veracruz (2020): "Veracruz Educando a Distancia", donde se publicaron guías complementarias, y el uso de la estación de radio oficial del estado (Radio-Televisión de Veracruz). En ninguno de los casos se encontraron elementos para la educación superior, ni tampoco para las instituciones formadoras de docentes.

Por lo anterior, ante esta ruptura con la normalidad, la UPV emitió tres documentos de política pública educativa predecisional al interior de la Universidad, para atender la emergencia académica derivada del confinamiento social:

a) Estrategia Académica para la atención de los estudiantes en el período de contingencia. Documento publicado ante la medida de "distanciamiento social", atendiendo a la información emitida por la Secretaría de Educación de Veracruz, en aras de salvaguardar la integridad de la comunidad universitaria con medidas para la atención de los Programas Académicos vigentes;

b) Estrategia Académica para los programas de estudio de Licenciatura y Posgrado ante la contingencia sanitaria COVID-19. Fase dos. A partir del anuncio de la suspensión de sesiones presenciales, la UPV implementó una estrategia académica, a través de la cual se transitó hacia los entornos virtuales de aprendizaje, por lo que se consideró construir un escenario a mediano plazo que permita atender la contingencia con una proyección al término del cuatrimestre y semestre.

c) Estrategia Académica para los programas de estudio de Licenciatura y Posgrado ante la contingencia sanitaria COVID-19. Fase dos y tres. Elementos para atender los programas educativos de la UPV de marzo a junio de 2020.

Aunado a lo anterior, es preciso considerar que la UPV cuenta con un modelo educativo vigente, denominado Horizonte Educativo (Velasco et al., 2016: 91-117), fundamentado en el aprender en el aprender y cuya tesis establece que para la formación inicial de los docentes se requiere incorporar elementos como la autorganización, la autonomía, la creatividad, la comunicación, el lenguaje, la intersubjetividad (trabajo colaborativo), la ética, la interculturalidad y las Tecnologías de Información y Comunicación (TIC). Además, involucra la mediación pedagógica que invita a "desarrollar el pensamiento divergente [...] esencial en el proceso de aprender en el aprender, lo que coadyuva a transitar de la ausencia de la emergencia a un comportamiento efectivo, permanente" (Velasco et al., 2016: 139). Es una propuesta pedagógica para la UPV que en su contenido expresa la necesidad de realizar un giro pedagógico.

En este sentido, y ajustando esta problemática a contextos locales, nos preguntamos ¿quiénes son los estudiantes de la UPV y cómo están pasando la contingencia sanitaria derivada del COVID-19? Para dar respuesta a este cuestionamiento se diseñó e implementó una estrategia de 
investigación que pone el acento en el estudiantado (entendido como sujeto actor educativo); todo ello, a partir de un enfoque multimetódico: cuestionarios en línea, registros de campo y observaciones en clases virtuales, así como entrevistas semiestructuradas. Todos los participantes formaron parte de los grupos de cada uno de los investigadores que integran el Departamento de Investigación de la UPV que en ese momento de crisis se encontraban dando curso, ya sea de nivel licenciatura, maestría o doctorado.

De esta manera pudimos dar cuenta de las estrategias, motivaciones y acciones que los estudiantes desarrollaron durante los primeros meses del confinamiento social al que orilló la contingencia sanitaria, y que hemos denominado ruptura con la normalidad, en el sentido de que implicó un cambio en las estrategias de enseñanza y de administración escolar a la Universidad Pedagógica Veracruzana, a diferencia de otras instituciones de educación básica y media superior que tenían claramente definido el rumbo de acción ante la emergencia.

Siendo así, a partir de las anteriores conceptualizaciones, nos dimos a la tarea de analizar quiénes son y cómo están pasando la contingencia sanitaria nuestros estudiantes de la UPV, en una temporalidad que abarca desde el momento de confinamiento social (o ruptura con la normalidad) hasta las primeras acciones realizadas ya en aislamiento, lo que implica un análisis inicial de políticas públicas posdecisión desde la perspectiva de su aplicación e interpretación pues cabe señalar que el estudio es más amplio y culminará con el regreso de los estudiantes a las aulas de la UPV, que seguramente podría tener una vertiente de evaluación en otro momento.

Por tanto, en este análisis nos dispusimos a realizar una serie de indagaciones sobre la situación que imperó. Nos preocupaba que las características del estudiantado de la UPV no fueran del todo tomadas en cuenta; esto, no como una crítica sin sentido, sino como una forma de apoyar futuras decisiones al respecto e incluso, que muchas estrategias surgidas en este contexto pudieran perdurar debido a su eficacia en los procesos de aprendizaje de los estudiantes.

\section{Enfoque teórico-conceptual}

Tomando en cuenta lo anterior, un grupo multi e interdisciplinario del SISE nos dimos a la tarea de conformar un enfoque teórico-conceptual que permitiera dar cuenta de las distintas acciones de los estudiantes en esta etapa de confinamiento sanitario, que en este trabajo se remonta a la vertiente antropológica del análisis institucional y de política pública.

Para ello se retomaron los planteamientos de la Antropología Crítica de la Acción (ACA), cuyo máximo exponente es Bensa $(2015,2016)$; del análisis institucional se retoman los planteamientos de Remedi (2008) y Fuentes $(2010,2012)$, y respecto al análisis de política pública se retoma a Parsons (2017), Lasswell (1992), Aguilar (1992, 2003), Fuentes (2018) y Villarreal (2010).

La intención fue posar la mirada en las construcciones simbólico-imaginarias elaboradas por los estudiantes (entendidos como sujetos actores educativos), lo que permitirá dar a conocer de manera interpretativa la situación desde sus propias voces.

Diálo@os sobre Educación año 12 | número 22 | enero-junio 2021 | ISSN 2007-2171 
De esta manera, se pretende resaltar la acción del sujeto, muchas veces olvidada u oculta tras el poder estructural de las políticas o por la propia dinámica que imprime la vida cotidiana, pues si bien las instituciones se encuentran reguladas por políticas -en este caso educativas-, estas no lo son todo al existir siempre un espacio para que los sujetos que las habitan coloquen sus deseos personales y desarrollar estrategias para alcanzarlos, y que no siempre concuerdan con lo señalado por las políticas.

Con lo que aquí se presenta no solo se intenta superar el punto de vista normativo, sino también la falacia racionalista y funcionalista (Majone, 2014) con la que tradicionalmente se ha abordado el estudio de las políticas, en las cuales se asume la tarea como un asunto meramente pragmático y que, en palabras de Fuentes-Amaya (2010: 10): "Parecen estar ausentes los sujetos educativos [...]; o, en todo caso, éstos son concebidos como entes predominantemente racionales cuyo resultado es producto de un cálculo que evalúa costos y beneficios y deja de lado aristas fundamentales como las construcciones simbólicas e imaginarias".

Por ello, aquí entendemos al sujeto no como mero efecto de la estructura o un ente que reacciona mecánicamente a las indicaciones o estímulos exteriores a él, sino que es "una criatura de emociones, por lo menos tanto como de razón" - palabras que James Frazer dirigió a Malinowski respecto de cómo este último describía a los nativos que estudiaba [...] (Jacorzynski, 2004: 75). Siendo así, en este trabajo no coincidimos con la idea de un actor determinado por un orden funcional o estructural (Bensa, 2016), sino que, al interior de las instituciones y al margen de las políticas, existen "sujetos activos que persiguen fines concretos [...], que experimentan medios distintos para alcanzarlos, que buscan el reconocimiento social, que dudan y reflexionan críticamente sobre sus errores y aciertos" (Viqueira, 2016: 7).

Las nociones de política, las políticas y lo político proceden de la palabra política. Dice Fuentes (2018) que se recurre a política(o) para nombrar múltiples cosas y usos:

Con la palabra política se han sustantivado múltiples aspectos de la actividad humana: se utiliza para nombrar y para adjetivar, para semantizar algo a la vez que para significarlo. Política puede ocuparse para referir a la acción de un actor político y también para nombrar a la persona que ejerce la función pública, por ejemplo. Con el término política, desde el sentido común, se sustantivan y adjetivan múltiples sujetos, objetos, procesos y prácticas de naturaleza variada (Fuentes, 2018: 34-35).

Por lo que política resulta ser el término más socializado en el espacio público para referir las acciones, los procesos y actores de la esfera de gobierno. En términos generales, como lo expresa Aguilar (2003), la política pública es un:

[...] proceso que se desenvuelve por etapas, cada una de las cuales posee sus actores, restricciones, decisiones, desarrollos y resultados propios; por lo que, influye en las demás y es afectada por lo que 
sucede en las otras políticas. Las etapas del proceso son componentes lógicos e interdependientes y la separación analítica no debe confundirse con una separación real o una secuencia temporal (Aguilar:

2003: 15-21).

Desde otra perspectiva, Lasswell (en Parsons, 2007: 32) precisa que "una política pública se distingue por ser metódica, multidisciplinaria, orientada a los problemas, interesada en el trazo del mapa conceptual del proceso, opciones y resultados de las políticas públicas e integradora de conocimientos". Por lo que el análisis de las políticas públicas tiene dos orientaciones: el estudio del proceso de las políticas y el estudio del conocimiento en el proceso de las políticas (Parsons, 2007; Aguilar, 2003).

En función de lo anterior, Villarreal (2010) identifica un ciclo de políticas públicas: I. Identificación de un problema público; II. Formulación de soluciones; III. Toma de decisión; IV. Implementación; V. Evaluación. Esto muestra que el análisis de estas puede tener dos grandes vertientes: el insumo de su creación (predecisión, que implica la identificación del problema público, la organización, entre otros) y la revisión de sus resultados (posdecisión, como la aplicación, interpretación, evaluación, entre otros) (Parsons, 2017: 53-59).

\section{Nota metodológica}

Como bien se anunció líneas arriba, lo que aquí presentamos corresponde a la primera fase de una investigación más amplia, la cual consiste en el seguimiento puntual y profundo de las acciones que los estudiantes de la UPV realizan en torno a sus experiencias educativas como parte de los programas educativos en los que se encuentren inscritos (licenciatura, maestría y doctorado) y de los cuales los miembros del SISE somos docentes. Para ello se ha utilizado el denominado Enfoque Multimétodo (EMM) que, en palabras de Ruiz-Bolívar (2008: 8), se caracteriza "por la implementación de dos o más procedimientos para la indagación sobre un mismo fenómeno u objeto de estudio a través de los diferentes momentos del proceso de investigación".

Por tanto, se utilizaron recursos provenientes tanto del paradigma cualitativo como cuantitativo ya que la combinación de distintas estrategias metodológicas permitió recolectar diferentes tipos de datos. El trabajo de investigación responde a un EMM secuencial interparadigmático ya que, aunque cada estrategia se utilizó de manera independiente, responden a la misma orientación epistémica para su posterior interpretación en los resultados mediante el procedimiento de triangulación (Ruiz-Bolívar, 2008).

Es así que se consideró a la etnografía como el enfoque central de acercamiento a los actores, en tanto que "permite dar cuenta de algunos aspectos de las vidas de unas personas sin perder de vista cómo éstas entienden tales aspectos de su mundo" (Restrepo, 2016: 16), pues el interés es mostrar quiénes son y cómo están pasando la contingencia sanitaria; todo esto nos resultó importante -en términos del análisis institucional y de política pública (siguiendo

Diálo@os sobre Educación año 12 | número 22 | enero-junio 2021 | ISSN 2007-2171 
a nuestro enfoque teórico-conceptual que coloca la mirada en el sujeto)- para comprender la acción de los estudiantes y poder plantear un horizonte prospectivo para la propia UPV.

De esta manera se logró realizar un trabajo de tipo descriptivo-interpretativo al conjugan datos sobre los sujetos y sus condiciones de estudio con la interpretación de dichas condiciones respecto a la política planteada por la institución para hacer frente a las estrategias, motivaciones y prácticas académicas ante el aislamiento social derivado de la pandemia.

Ahora bien, decidimos que la muestra para el estudio fueran nuestros propios estudiantes (en tanto que como personal activo de la universidad nos encontrábamos frente a grupo dando clases en distintos niveles educativos); por lo que la muestra (denominada por conveniencia) se conformó por un total de 82 estudiantes de los niveles de licenciatura, maestría y doctorado, ubicados en los Centros Regionales de Estudios (CRE) de Xalapa, Veracruz, Orizaba, Córdoba y Minatitlán. ${ }^{1}$

Respecto a la recuperación de insumos, primero se elaboró un instrumento para dar cuenta de esta primera etapa, Ruptura con la normalidad, que consistió en una encuesta configurada por tres ejes específicos, a saber: 1) Generalidades de los actores, 2) Dimensión institucional, y 3) Opinión de los actores. La encuesta se aplicó en línea (vía Google Forms). ${ }^{2}$

Aunado a lo anterior, los integrantes del SISE, al momento de impartir las clases, realizamos un registro de campo en el que fuimos anotando parte de las experiencias de nuestros estudiantes, las cuales eran discutidas durante las sesiones. Otra de las técnicas implementadas fue la realización de entrevistas semiestructuradas, con las que fuimos explorando nuestros registros de campo.

Así, estuvimos en posibilidad de dar cuenta de las acciones institucionales, pero también de los estudiantes, y describir sus estrategias y motivaciones de continuar sus estudios durante la pandemia.

\section{Descripción de los datos}

El presente apartado se estructura con base en las tres dimensiones arriba señaladas, tratando de responder a la pregunta guía de este trabajo: ¿cómo están pasando la contingencia sanitaria los estudiantes de la UPV? Pero esta pregunta no se puede responder sin antes preguntarnos: ¿quiénes son esos estudiantes?

Lo anterior resulta altamente relevante no solo en su dimensión heurística, sino para la propia UPV, ya que a pesar de ser una institución con casi 40 años de servicio y 15 sedes (cono-

1 Cabe hacer mención que la UPV cuenta con 15 Centros Regionales de Estudios a lo largo del estado de Veracruz.

2 Con la primera dimensión (Generalidades) se buscó dar cuenta de quiénes eran nuestros estudiantes, dónde se ubicaban y las condiciones con las que pasan la contingencia sanitaria. La segunda dimensión (Institucional), se buscó conocer todas aquellas acciones que nos parecían importantes que la UPV hiciera a favor de los estudiantes, como: capacitación docente y de estudiantes, así como la implementación de alguna plataforma. Con la tercera dimensión (Opinión), quisimos dar cuenta de las percepciones y acciones que los estudiantes construían respecto de la situación y sus estudios. 
cidas como Centros Regionales de Estudios), en el estado de Veracruz poco se sabe acerca de sus estudiantes y sus condiciones de estudio. Ante esta situación, la relevancia de este trabajo es, por así decirlo, doble: por un lado, el universo de conocimiento sobre los estudiantes de la UPV se ampliará y profundizará; y por otra, la propia institución tendrá elementos para plantear un horizonte educativo que incorpore el conocimiento aquí construido a partir de las vivencias de los actores educativos.

\section{1) Generalidades de los actores}

Lo primero que hay que señalar es que del total de la muestra, 62 fueron mujeres (76\%) y 20 varones (24\%), cuyas edades van desde 18 a más de 40 años; de estas: 18 personas se encuentran en el rango de 18-21 años, siete entre 22-25 años, 18 de 26-30 años, 14 entre 31-35 años, 8 de 36-40 años y 17 con más de 40 años (figura 1).

Figura 1. Edad de los aprendientes que integran la muestra

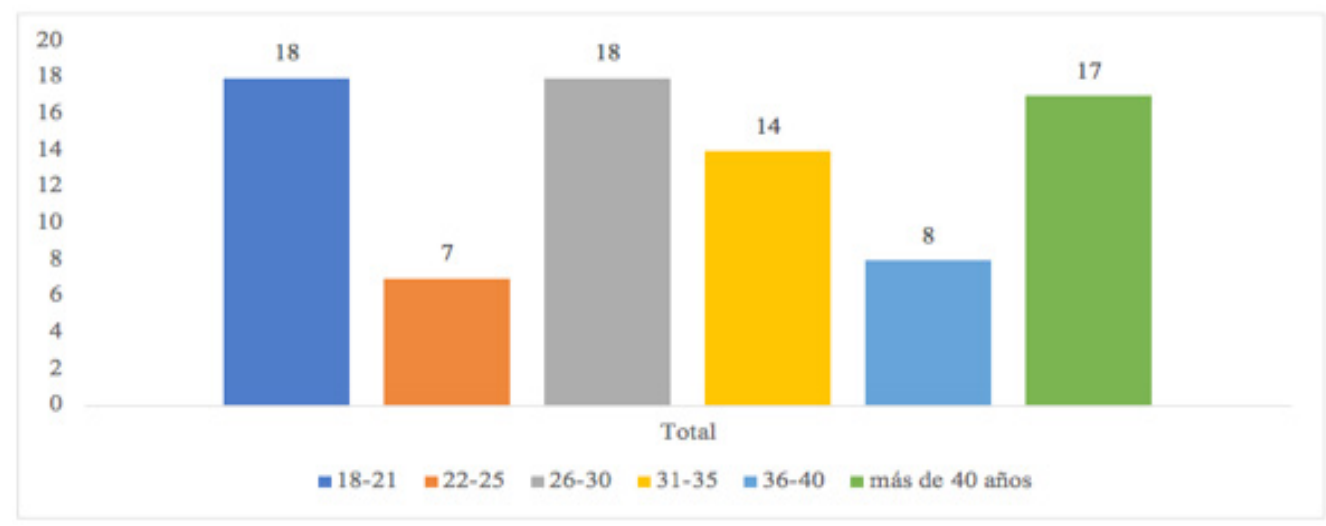

Fuente: Base de datos integrada por el SISE.

En cuanto al CRE en el que se encuentran, los datos arrojaron que 56 estudiantes se ubican en Xalapa, 12 en Minatitlán, ocho en Orizaba, cuatro en Veracruz y dos en Córdoba (figura 2); los cuales son procedentes de 29 municipios del estado de Veracruz, ubicados en distintas zonas escolares. 
Figura 2. Distribución por Centro Regional de los aprendientes que integran la muestra

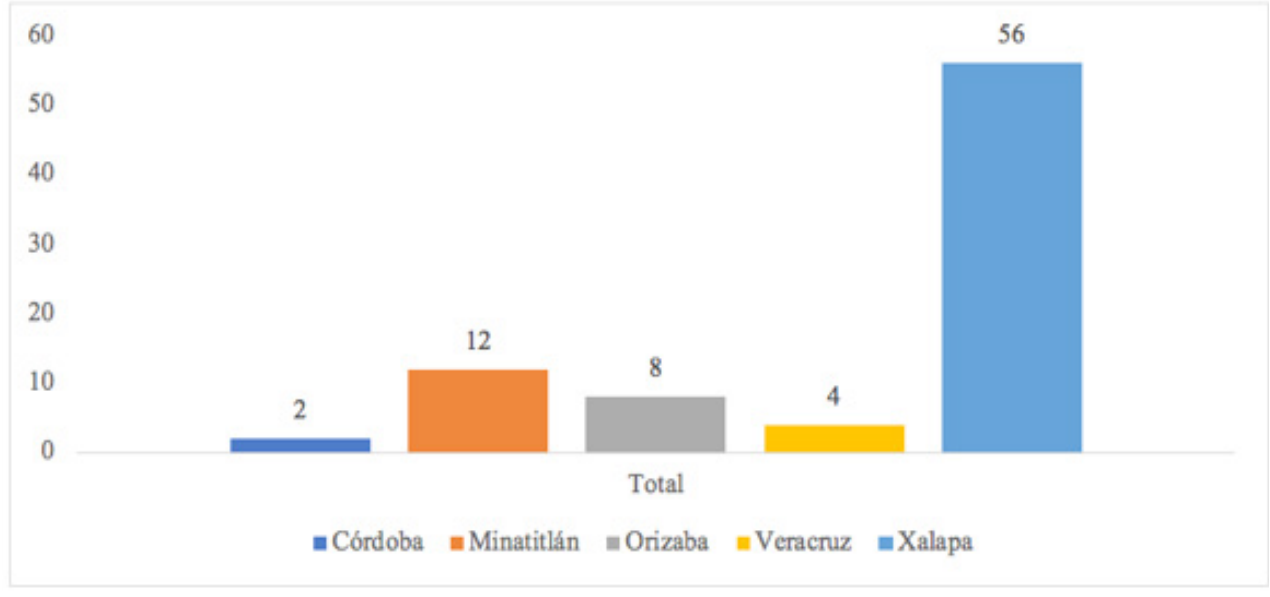

Fuente: Base de datos integrada por el SISE.

En cuanto a su actividad, 33 personas (40\%) refirieron ser docentes frente a grupo en distintos grados y niveles; estos van desde preescolar (17\%) hasta el nivel superior (6\%), siendo el grado de primaria donde se ubica el mayor porcentaje (46\%) (figura 3).

Figura 3. Estudiantes de la UPV que son docentes frente a grupo

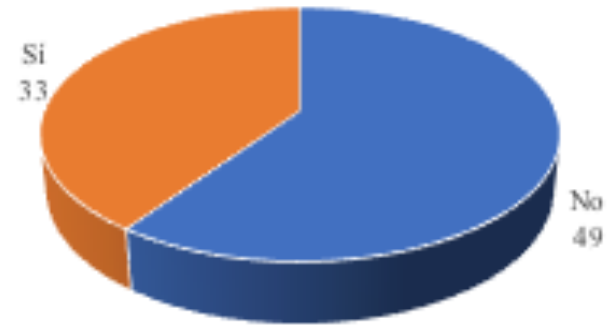

Fuente: Base de datos integrada por el SISE.

Ahora bien, de los 33 estudiantes de la UPV que son maestros frente a grupo, $63 \%$ continuaron sus actividades docentes de manera virtual durante esta contingencia sanitaria y $37 \%$ no la están realizando en estos momentos (figura 4). 
Figura 4. Estudiantes de la UPV que continuaron actividades de manera virtual

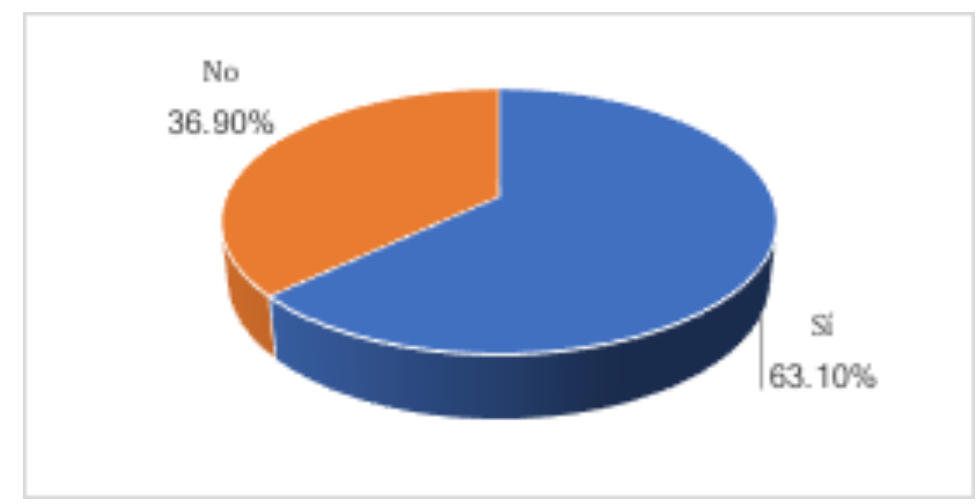

Fuente: Base de datos integrada por el SISE.

De los 33 estudiantes docentes frente a grupo, 23 están cursando la maestría, cinco el doctorado y otros cinco la licenciatura (figura 5). Respecto a su género, 22 son mujeres y 11 varones.

Figura 5. Distribución de estudiantes por nivel de estudios en la UPV, que son docentes frente a grupo (en relación con $63 \%$ que continuó con actividades de docencia)

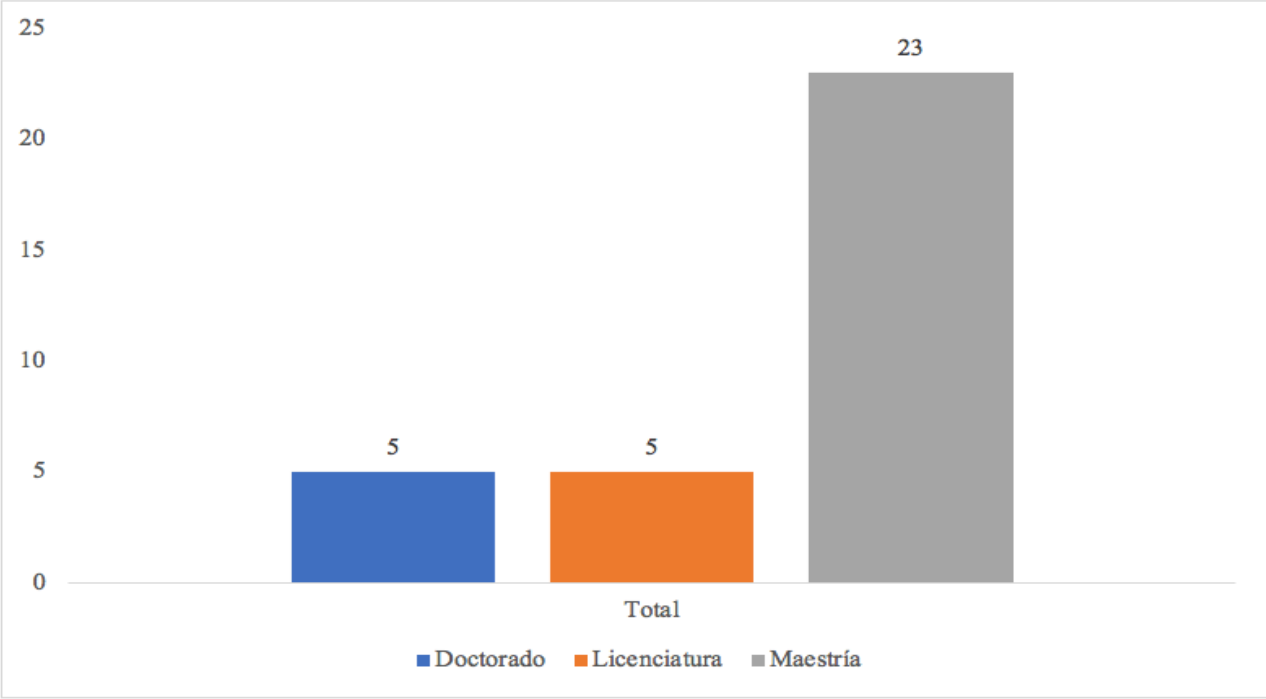

Fuente: Base de datos integrada por el SISE.

Respecto a cómo están pasando la emergencia sanitaria (quédate en casa), 89\% lo hace en su residencia habitual y $11 \%$ en otra residencia; de los 82 estudiantes de la muestra, 25 personas 
viven con tres personas más, 15 con una persona, 14 con cuatro, 12 con dos, 8 con cinco y otras 8 con más de cinco personas (figura 6).

Figura 6. Con cuántas personas están pasando la emergencia sanitaria

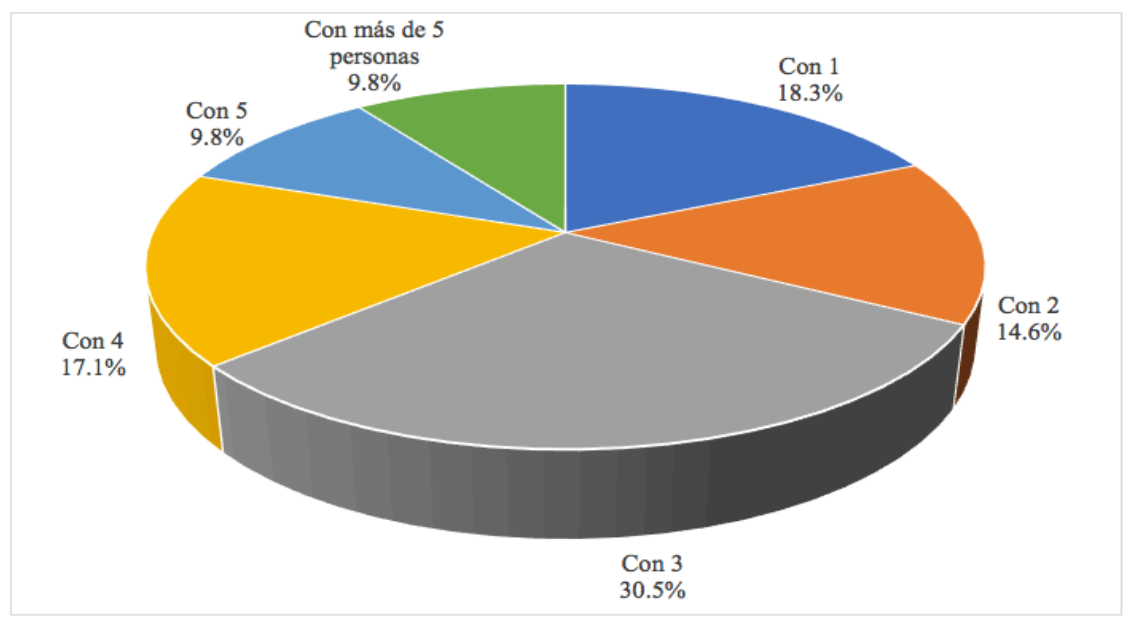

Fuente: Base de datos integrada por el SISE.

El dato anterior lo hemos desagregado por género, lo cual se muestra en las figuras 7 y 8.

Figura 7. Mujeres. Con cuántas personas están pasando la emergencia sanitaria

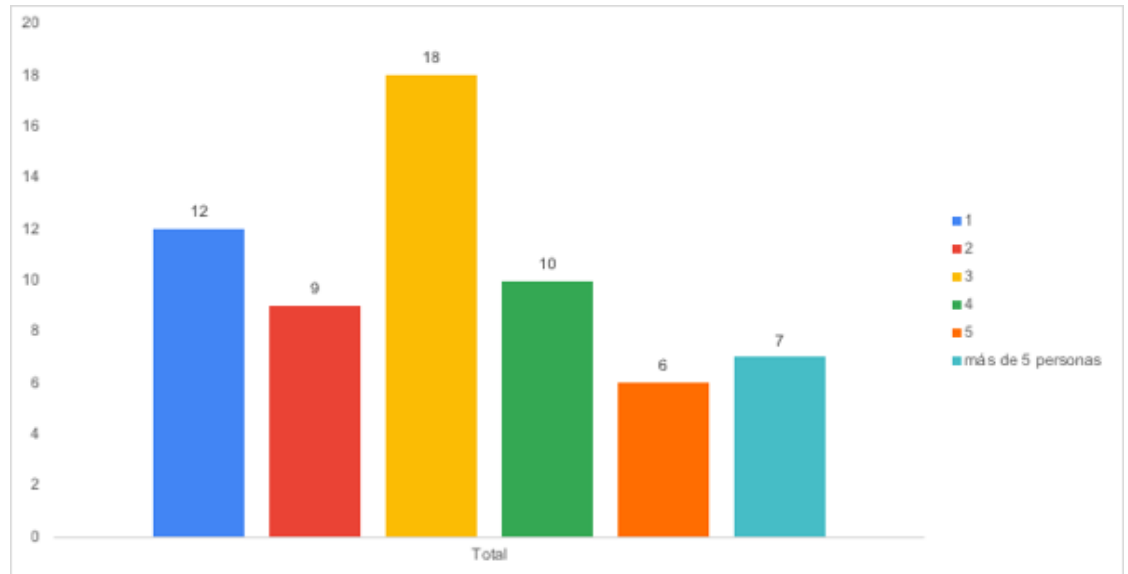

Fuente: Base de datos integrada por el SISE. 
Figura 8. Varones. Con cuántas personas están pasando la emergencia sanitaria

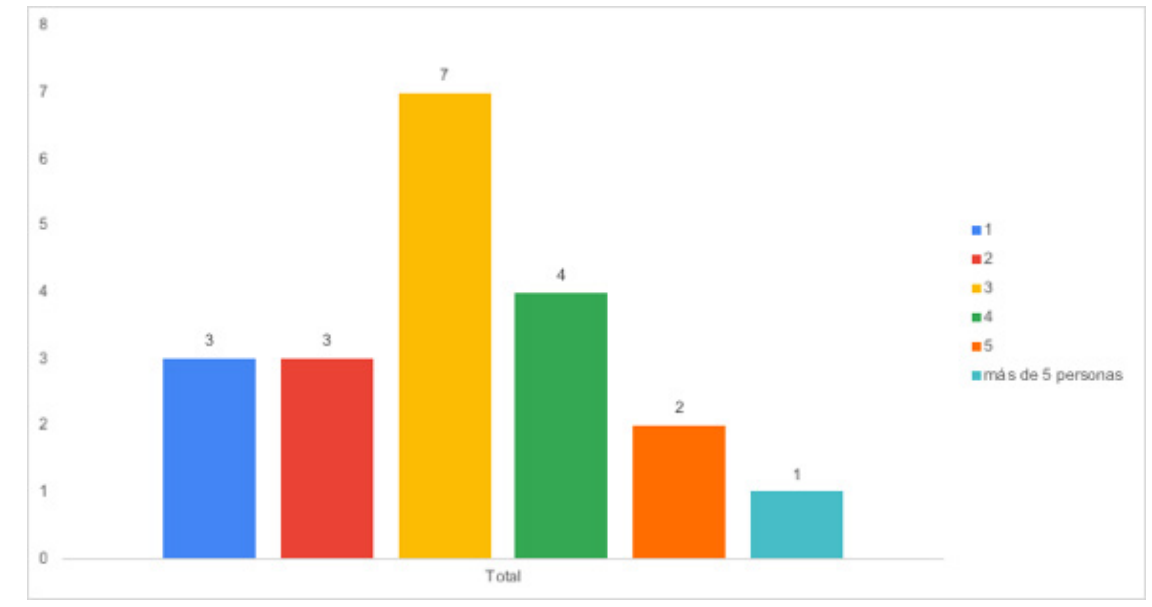

Fuente: Base de datos integrada por el SISE.

Del total de la muestra, 33 personas (40\%) refirieron tener hijos en edad escolar que toman clases virtuales (vía internet o televisión) (figura 9). De los cuales, 25 son mujeres y 8 varones; además, 26 de ellas mencionaron que tuvieron que adquirir un equipo para ello y 28 tuvieron que contratar algún servicio de internet.

Figura 9. Personas que tienen hijos en edad escolar

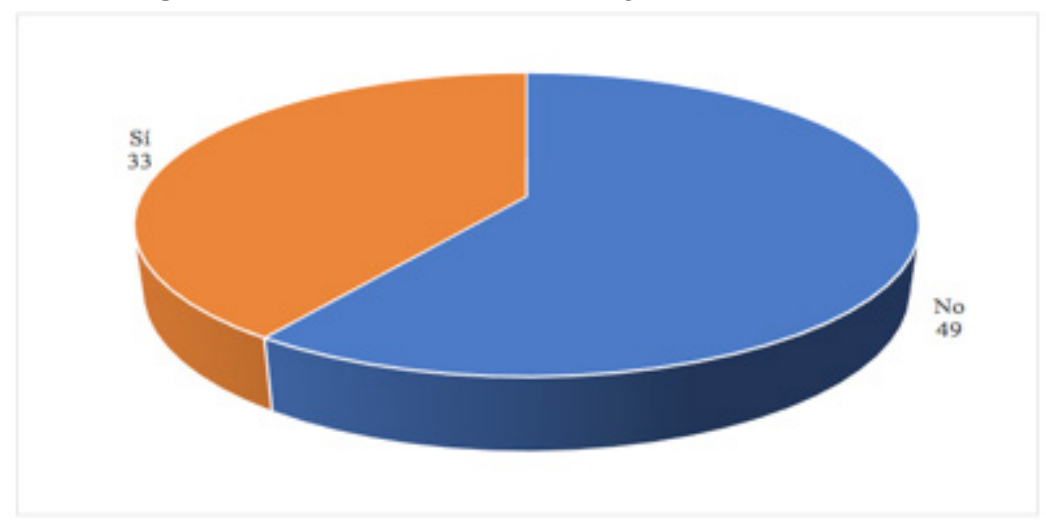

Fuente: Base de datos integrada por el SISE.

\section{2) Análisis de la dimensión institucional}

Pasando a una dimensión institucional, es decir, lo que la propia UPV ofreció al momento de la emergencia sanitaria, las referencias al señalamiento de una plataforma institucional para el de- 
sarrollo de las actividades académicas están divididas: 49\% mencionó que no lo hubo, mientras que $51 \%$ que sí; la mayoría de las respuestas afirmativas provienen de estudiantes del nivel de maestría (figura 10).

Figura 10. Desde la UPV, le han señalado una plataforma institucional para el desarrollo de las actividades académicas

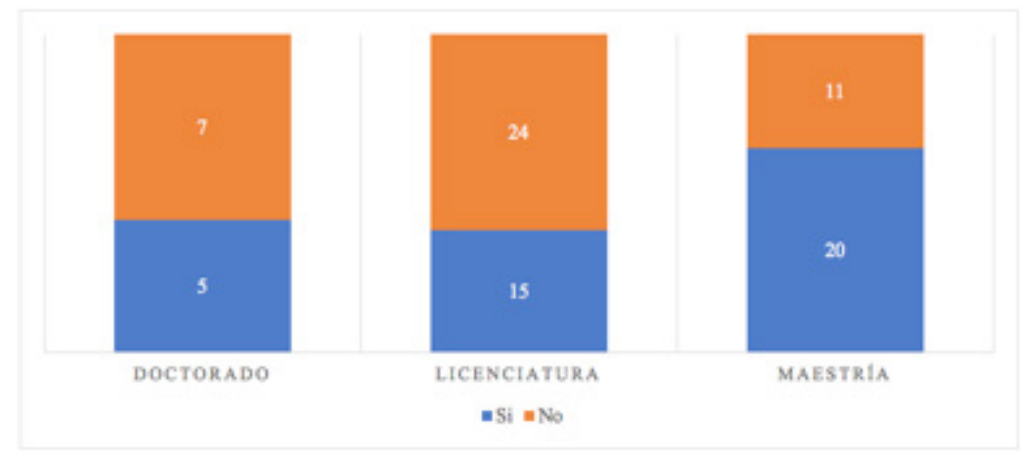

Fuente: Base de datos integrada por el SISE.

Al respecto, evidentemente hubo confusión por parte de los participantes ya que la UPV no cuenta con una plataforma institucional, por lo que hicieron uso de plataformas comerciales como Zoom (donde 83\% están desarrollando sus actividades de clases virtuales), Classroom y Hangouts.

Otro aspecto a destacar es que $68 \%$ de los participantes mencionaron que no recibieron ningún tipo de capacitación para el uso de plataformas (SISE, 2020), lo cual indica que la respuesta de la UPV ante la emergencia sanitaria no contempló las condiciones de los estudiantes; además de que muchos tuvieron que compartir equipos de cómputo y contratar servicios de internet para poder realizar las actividades.

\section{3) Opinión de los actores}

En cuanto al desarrollo de las actividades académicas durante la emergencia sanitaria, de las 82 personas que conformaron la muestra, 29 mencionaron que las desarrollaron muy bien, 24 bien, 17 regular, 8 mal y 4 muy mal (figura 11). 
Figura 11: Los alumnos ¿cómo creen que se han desarrollado las actividades académicas?

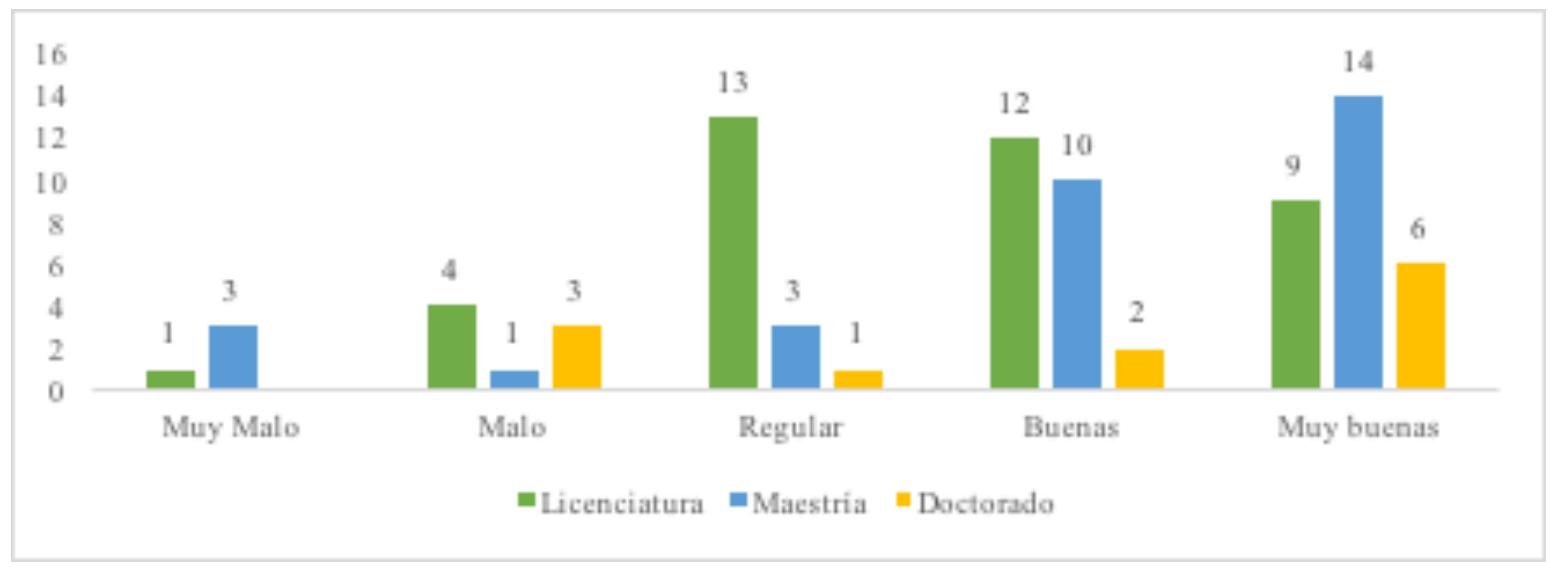

Fuente: Base de datos integrada por el SISE.

Lo anterior contrasta un poco con la opinión de los propios estudiantes respecto a su percepción sobre si los docentes de la UPV recibieron capacitación para la planeación y desarrollo de sus actividades académicas de forma virtual, pues existe un alto grado de incertidumbre; 38 personas refirieron que tal vez, 14 que no y 30 que sí (figura 12).

Figura 12: Los alumnos ¿consideran que los contenidos curriculares se están alcanzando de forma correcta?

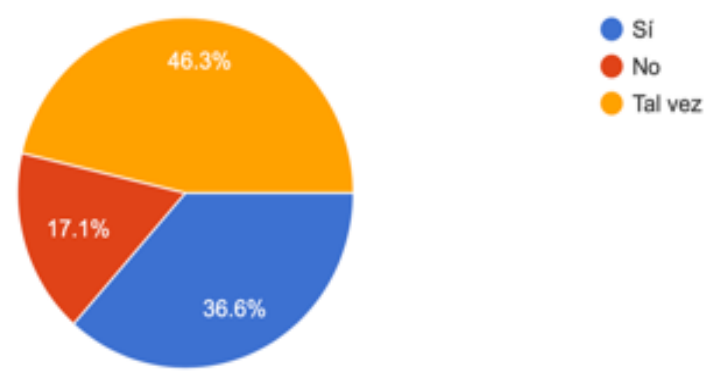

Fuente: Base de datos integrada por el SISE.

En cuanto a los propios estudiantes, 56 (68\%) mencionaron que no recibieron capacitación para el uso y manejo de plataformas virtuales; aunque esto no afectó su percepción sobre alcanzar de manera correcta los contenidos curriculares, donde $67 \%$ mencionó que sí lo están haciendo (figura 13). 
Figura 13: Considera que los docentes de la UPV recibieron capacitación para la planeación y desarrollo de sus actividades académicas

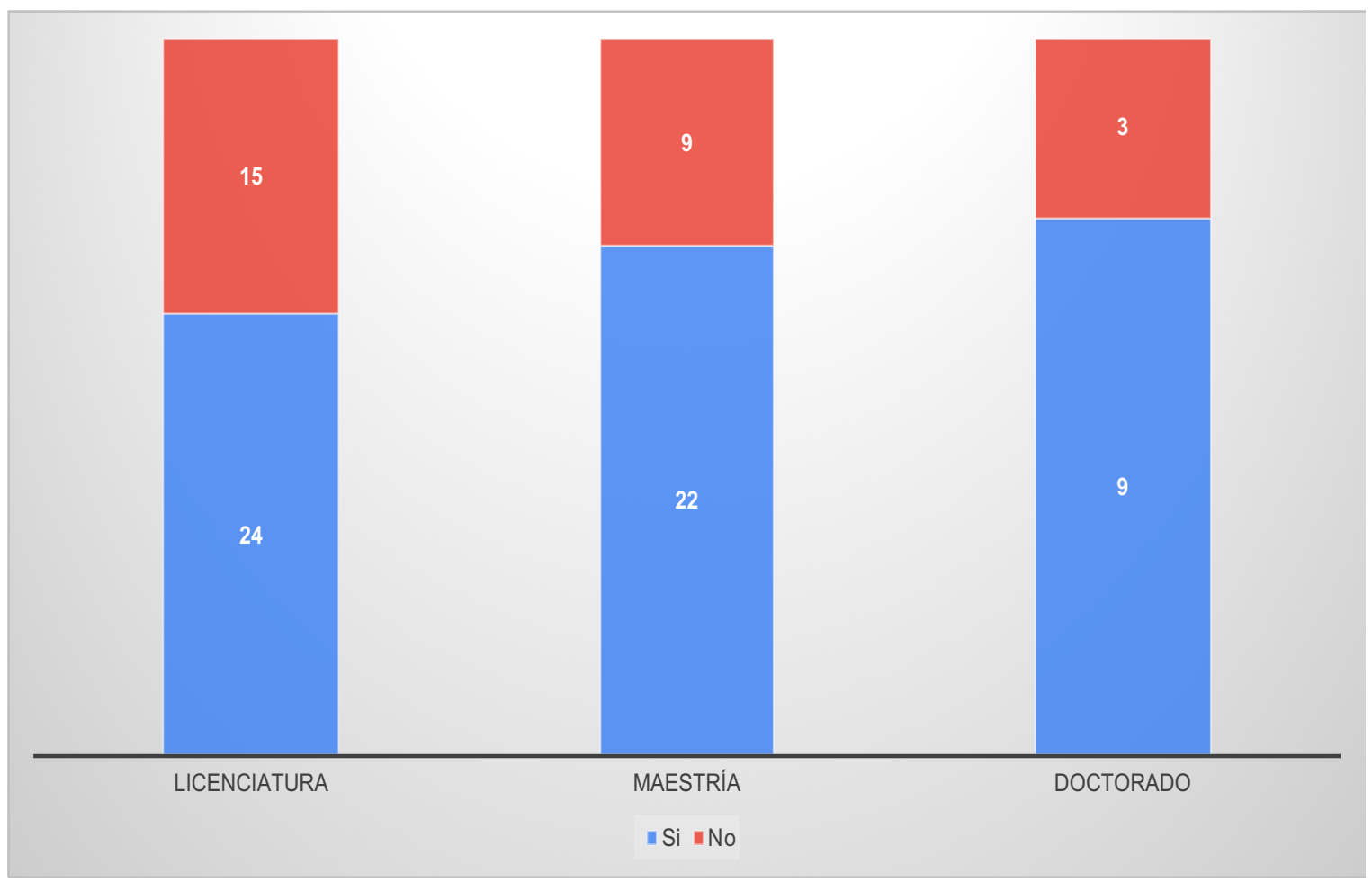

Fuente: Base de datos integrada por el SISE.

Así, desde la opinión de los estudiantes de la muestra, 29 mencionaron que el desarrollo de sus actividades va bien, 27 que muy bien, 18 regular, 4 mal y 4 muy mal. Además de que 32 personas no saben si esta situación afectará su desarrollo académico, 23 que sí lo hará y 27 que no. En términos generales, $61 \%$ de la muestra refirió que el contexto actual derivado de la contingencia sanitaria no les ha permitido concentrarse más en sus estudios. 
Figura 14: Consideras que esta emergencia sanitaria te ha permitido concentrarte más en tus estudios de la UPV

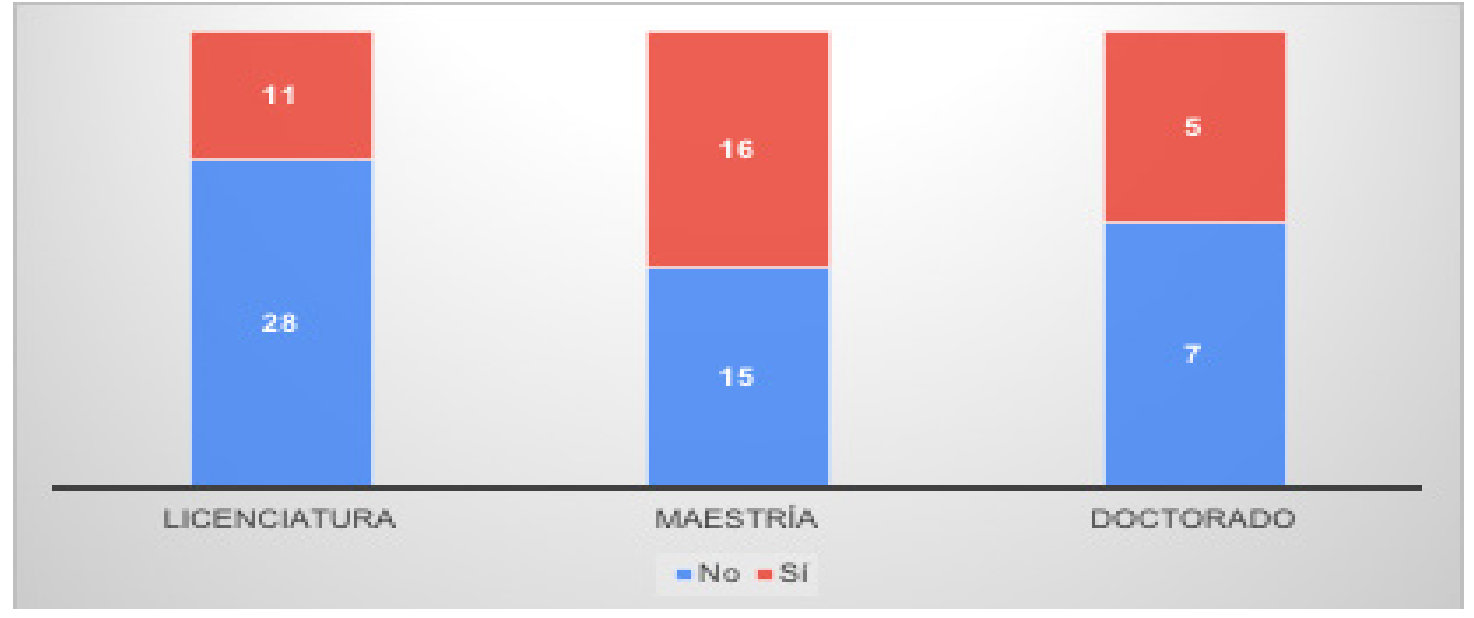

Fuente: Base de datos integrada por el SISE.

Aunado a lo anterior, y derivado del análisis de los registro de campo y las entrevistas, se encontró que una de las principales estrategias implementadas por los estudiantes de la UPV es la autogestión y organización de las actividades, como lo establece el Horizonte Educativo de la universidad, que consiste en la organización del tiempo de estudio con las actividades cotidianas durante el confinamiento; como se pudo ver en el apartado anterior, muchos de ellos comparten equipos y además atienden asuntos familiares, por ello, han tenido que autogestionar sus tiempos para la organización de todas sus actividades. Así, ante estas situaciones han tenido que jerarquizarlas y establecer horarios para el desarrollo de todas las actividades (no solo estudiantiles).

Otra estrategia de autogestión y organización de las actividades ha sido el mantenerse en contacto con compañeros y maestros mediante el uso de herramientas tecnológicas (plataformas virtuales, aplicaciones como WhatsApp o Skype).

Punto central de esta estrategia ha sido el denominado aprendizaje autónomo. Al respecto mencionaron: elegir un lugar y tiempo adecuados para el trabajo escolar; tener lista las aplicaciones para dialogar con compañeros; muchos, por ejemplo, realizan "resúmenes, esquemas, mapas mentales, foros de debate en línea con mis compañeros" (Opinión_Estudiante 54, 2020), otros realizan "ejercicios autónomos de búsqueda de información, elaboración de mapas conceptuales" (Opinión_Estudiante 24, 2020).

Otra de las estrategias implementadas ha sido la diversificación de actividades. Aquí, en tanto que los estudiantes no son solo eso, sino que también son padres o madres, maestros, o bien comparten el espacio de confinamiento social con otras personas, quisimos dar cuenta 
de sus estrategias. Por ejemplo, refieren: "realizar mis actividades por la noche, para poder hacer mis actividades como mamá durante el día" (Opinión_Estudiante 77, 2020), o bien "organizar mis tiempos entre la casa, mi hijo y la escuela" (Opinión_Estudiante 33, 2020). Esto los ha llevado a programar horarios y actividades.

La última estrategia es la que hemos denominado sobreposición de actividades presenciales, esto es, pensar que las actividades son tal cual fueran en la escuela. Aquí refieren "hacer de cuenta que sigo en clases normales, ya que solo asistía los sábados. Además, sigo solo las instrucciones de las actividades" (Opinión_Estudiante 82, 2020).

Así, muchos mencionan que solo siguen al pie de la letra las instrucciones que los profesores emiten en las plataformas virtuales para el cumplimiento de las actividades estudiantiles, por lo que solo en el horario establecido se dedican al estudio, por ejemplo: "Ios sábados los destino a mis clases en línea como si estuviera de forma presencial" (Opinión_Estudiante 42, 2020). Por tanto, una de las principales acciones dentro de esta estrategia es seguir las instrucciones que los docentes establecen para el cumplimiento de las actividades escolares.

En lo que se refiere a las motivaciones de los estudiantes, las hemos agrupado en las siguientes categorías: aprendizaje personal, profesión e ingresos, cumplimiento de metas y familia. Dentro de la primera se incluyen todas las referencias a la motivación de lograr un aprendizaje que les permita mejorar sus prácticas. Así, refieren que continuar con su formación para lograr un aprendizaje es una forma de superación personal, como lo mencionan: "las ganas de superación" (Opinión_Estudiante 02, 2020). Por tanto, continuar con las actividades académicas es una forma de superación personal en la que el aprendizaje adquirido es significado, como "el deseo de ser docente, de aprender más cosas y de aprender estrategias para un aprendizaje significativo" (Opinión_Estudiante 67, 2020).

Otras dos motivaciones mencionadas por los estudiantes se refieren a la mejora económica y el cumplimiento de una meta establecida (culminar los estudios), formuladas antes del confinamiento; así, mencionan "las ganas de salir adelante y terminar con éxito lo que empecé" (Opinión_Estudiante 73, 2020) o bien "los beneficios que pudiera derivar de ello [culminar los estudios]" (Opinión_Estudiante 23, 2020).

Por último, la motivación más mencionada fue la relacionada con la familia y el apoyo que reciben por el esfuerzo que los estudios requieren; refieren: "me motivan mis hijos, darles un ejemplo que a pesar de las cosas no hay que rendirse. Hay que continuar con el esfuerzo" (Opinión_Estudiante 44, 2020). En este sentido, encontramos que la familia es un punto de apoyo fundamental para los estudiantes de la UPV.

\section{Análisis de resultados}

Después de haber revisado los resultados de nuestro trabajo de investigación, fue posible observar que ante la contingencia sanitaria derivada de la pandemia de COVID-19 y el impedimen- 
to de realizar actividades sociales, como asistir a las escuelas, la UPV respondió de manera inmediata, incluso se publicaron lineamientos para el trabajo desde casa con la finalidad de alcanzar los aprendizajes plasmados en los programas de estudio.

Además, muestra que los documentos y acciones institucionales diseñados en la UPV parecen no tomar en cuenta los contextos personales y profesionales de los estudiantes, pues en la gran mayoría de los casos se trata de estudiantes que durante la semana laboran o bien tienen que atender otras actividades; esto se reflejó cuando la mayor parte de los estudiantes hicieron mención de que pasaron el tiempo de la contingencia sanitaria con al menos otra persona (algunos con sus hijos en edad escolar, que igualmente tenían que tomar sus cursos en línea, por lo que tuvieron que compartir sus equipos de cómputo).

Otro asunto que reflejó el análisis fue la necesidad de contratar algún servicio de internet para atender las actividades en línea, ya que antes de la pandemia (eso que hemos denominado ruptura con la normalidad) las clases en la UPV eran los días sábados de forma presencial, lo que les permitía atender otras actividades señaladas (como la docencia o el cuidado de sus hijos); esto implicó un gasto extra para muchos estudiantes, incluso, el aprender a usar las herramientas tecnológicas que se han implementado.

Por otra parte, en la dimensión institucional, se revisaron documentos emitidos por la Universidad para implementar una serie de acciones con la finalidad de que sus estudiantes pudieran continuar los estudios y, aunque eso no implicó una capacitación para los docentes ni para los estudiantes respecto al uso de las plataformas virtuales, los propios estudiantes refirieron que esta situación no afectará del todo el cumplimiento de los contenidos curriculares. De igual forma, señalaron una amplia aceptación a las acciones realizadas por los docentes, pero en las que también estuvieron involucradas las propias motivaciones de los estudiantes, lo cual, desde la perspectiva que hemos planteado, es un punto clave ya que nos muestra que muchas acciones instituciones no tienen un efecto por sí mismas, sino que se cumplen por la voluntad de los sujetos involucrados; son ellos que han decidido continuar con cierto ímpetu sus tareas de aprendizaje.

Lo anterior provocó, de manera no intencionada, que los estudiantes accionaran una serie de saberes en sus prácticas y procesos de aprendizaje (al igual que los docentes respecto a la enseñanza), pues la institución no brindó ningún tipo de capacitación para el uso de herramientas tecnológicas, por ejemplo, el uso de distintas plataformas y aplicaciones de comunicación para participar en las sesiones de clases virtuales. Es importante señalar que por parte de la institución no existió un señalamiento para el uso de una plataforma específica sino que dejó en manos de los propios maestros esta decisión, por lo que los estudiantes tuvieron que adaptarse a las especificaciones de cada maestro.

Aunado a lo anterior, otro tipo de saberes y prácticas desarrolladas por los estudiantes es en el sentido de la organización de sus actividades escolares combinándolas con las de su vida 
cotidiana o, en algunos casos, con personas que los acompañan en la contingencia (hijos, parejas y otros). A partir de los datos que arrojó el estudio y a pesar de las estrategias académicas empleadas, en las que sí fueron tomadas en cuenta las características del estudiantado de la UPV, la opinión de los estudiantes refleja un contexto personal y profesional adverso y complicado ya que muchos de ellos son profesionales que toman clases los sábados.

Todo lo anterior nos señala que en la UPV no se está realizando una educación a distancia propiamente, puesto que durante la emergencia sanitaria mostró que aún carece de los conceptos e instrumentos para llevar a cabo una educación en línea, aunque sí ha buscado resolver la situación a través de los instrumentos tecnológicos.

De esta forma, nos encontramos ante la posibilidad de plantear la idea de un sujeto-estudiante autoorganizado, que dadas las condiciones ha tenido que hacer uso de sus saberes o bien, irlos construyendo en la práctica; desde el propio esfuerzo de los estudiantes $-\mathrm{y}$, por supuesto, de los docentes-, quienes señalaron la motivación por culminar sus estudios como la mayor razón de continuar estudiando en las condiciones de contingencia sanitaria derivada del COVID-19.

A pesar de que se están haciendo esfuerzos mayúsculos por dar continuidad a los procesos educativos, es imperativo mencionar que el currículo presencial de la UPV se está trasladando para ser impartido a distancia, en una especie de sobreposición de las actividades presenciales, pero ahora en el ámbito virtual (dicho de otra forma, si un curso tiene sesiones presenciales durante nueve semanas, en línea se coloca la misma cantidad de sesiones virtuales, tratando de atender el mismo número de actividades). El asunto aquí es que esto no es suficiente pues, como se ha mencionado al considerar el contexto de los estudiantes, provoca que sientan que tienen más trabajo; por ello, la institución debe plantear un trabajo efectivo para el desarrollo de los aprendizajes en modalidad a distancia, lo que involucra una serie de planteamientos específicos para este tipo de educación.

\section{Conclusión en clave prospectiva}

Por todo lo señalado anteriormente, advertimos que si bien la UPV, con la finalidad de contrarrestar los efectos negativos derivados de la ruptura con la normalidad, accionó un plan de trabajo con la intención de convertir la crisis en oportunidad, las condiciones reales de sus estudiantes no fueron del todo tomadas en cuenta, y por lo tanto, las acciones quedaron en manos de los docentes y la voluntad de los propios estudiantes, quienes han logrado que la experiencia sea un tanto exitosa en términos de aprendizaje bajo las condiciones que esta situación nos plantea.

Esto nos invita a repensar la forma en que seguirá atendiéndose la crisis en el periodo de contingencia (en tanto que se ha extendido más allá de lo planteado en su inicio), para llevar a buen puerto los planes y programas de estudio de la Universidad desde dos grandes perspectivas: el docente y el alumno. Pues la UPV, a diferencia de otras instituciones de educación 
superior, no cuenta con una plataforma propia para el desarrollo de las actividades virtuales; sin embargo, ello no implica la imposibilidad de que se desarrollen con un mayor grado de sistematicidad y orden, indicando el uso de una plataforma única para las clases.

En este sentido, los documentos de política pública educativa emitidos por la UPV a fin de atender esta ruptura de la normalidad, presentaron elementos de regulación mínima: a los docentes les otorgaron la potestad de definir el entorno virtual de aprendizaje, realizar una planeación y evaluación, informar a los alumnos, generar el trabajo en línea, llevar a cabo una retroalimentación y hacer seguimiento. No obstante, estos lineamientos fueron atendidos por docentes y alumnos de manera elemental; es decir, solamente atendieron referentes empíricos inmediatos, con la meta de evitar la pérdida de aprendizaje con el uso de tecnología y el aumento de deserción escolar, proteger la salud y la seguridad de la comunidad escolar, entre otros aspectos.

Esto se ve reflejado en el uso de diversas plataformas: Zoom y Google Meets (para video conferencias) Google Classroom y Edmodo (como plataformas educativas) y, en último caso, el Whatsapp y correo electrónico (como medios de comunicación alternativos). En ninguno de los documentos se hizo mención a la plataforma educativa de la UPV. ${ }^{3}$

De igual forma, desde este análisis posdecisional y en el que hemos colocado la mirada en la acción del sujeto, se debe plantear un plan que contemple una capacitación de los docentes, pero también que tome en cuenta los contextos personales y profesionales de los estudiantes, así como plantear una revisión para trasladar lo presencial a lo virtual, considerando que existen diversas modalidades, sistemas y opciones educativas en México (abierta, no escolarizada, no presencial, a distancia, en línea o virtual).

Esto implica la búsqueda de un marco conceptual sobre la opción educativa a adoptar en la UPV. Si bien es cierto que la Licenciatura en Educación Básica que ofrece la UPV es un programa bimodal (por realizar trabajos autónomos entre semana y de manera presencial atender a los alumnos cada fin de semana), existen otros programas (maestría y doctorado) que cuentan con un fundamento académico únicamente presencial.

Otro punto que nos señala este trabajo de investigación está en relación con la construcción de los aprendizajes necesarios para el desarrollo de una profesión, donde es igualmente importante el cómo se construyen pues no se trata solo de consumir el currículo planteado por una institución, sino que el camino para la construcción de esos aprendizajes en la educación superior debe ser significativo para el alumnado; por lo que las estrategias de enseñanza deben adecuarse a las condiciones de los estudiantes.

3 La plataforma educativa de la UPV se encuentra en el sitio web: https://upvv.clavijero.edu.mx/login/y está alojada en los servidores informáticos institucionales de la Secretaría de Educación de Veracruz, bajo la coordinación del Consorcio Clavijero. Cabe destacar que en esta plataforma educativa solo está vigente la Licenciatura en Educación Básica, los demás programas educativos, en la actualidad, no cuentan con una plataforma educativa propia.

Diálo pos 
Con los elementos planteados en esta conclusión, es importante retomar la prospectiva de Miklos y Tello (2015), descrita como "preparar el camino, adaptándolo como objetivo (deseable y posible), es decir, guía las acciones presentes y el campo de lo posible del mañana." Además, encamina hacia los objetivos de: $a$ ) construir escenarios o imágenes; $b$ ) aportan elementos estratégicos; c) impulsa la planeación fundamentada en visión del futuro; y d) aporta una guía conceptual conductora del estudio.

Para Miklos, la prospectiva debe tener elementos básicos: visión holística (estudio de la influencia mutua entre las partes y el todo), creatividad (capacidad originar cosas nuevas y valiosas con el mejor modo de hacerlas), participación y cohesión (un objetivo común, con acuerdo sobre la problemática, analizando las capacidades y potencialidades, así como la decisión de cada actor), pertinencia (la unidad conceptual que hace posible que se insista menos en la coordinación explícita de las acciones), convergencia-divergencia (proceso iterativo con alto grado de complejidad), así como la finalidad constructora (¿hasta dónde llega?, respondiendo en dos aspectos: conceptual y práctico).

Para Baena (2015), a partir de los estudios de Deplhi sobre la previsión del futuro, la previsión se refleja como una espiral y la incertidumbre se refleja como un abanico debido a las varias direcciones que conforma. Para ella, la Planeación Prospectiva Estratégica es saber a dónde se tiene que llegar, acción en la que se determina el futuro deseado creativamente y libre de restricciones, y considera el pasado y el presente en un segundo momento (sin llegar a ser restricciones). Una vez que se visualiza la imagen del futuro deseado, se exploran los futuros factibles para seleccionar el más satisfactorio.

Lo anterior se señala en el marco del impacto mundial que ha sufrido la educación a consecuencia de la pandemia de COVID-19 ya que según el Banco Mundial (2020), hasta el mes de abril de 2020 se habían cerrado escuelas en 180 países y 85\% de los estudiantes del mundo habían dejado de asistir a la escuela; además de una crisis económica mundial de pronóstico reservado, pues calcula que el PIB global se retraerá $3 \%$ y tendrá costos a largo plazo en el sentido de que el abandono de la escuela por parte de los estudiantes generaría inequidad en los aprendizajes y pondría en riesgo a toda una generación de hacer realidad su verdadero potencial (Banco Mundial, 2020: 5-6).

Estos últimos elementos de prospectiva implican repensar o reconfigurar la UPV a fin de retomar el Horizonte Educativo (2016) para que pueda mostrar sus planes y programas de estudio desde una perspectiva o modalidad diferente. Generar una prospectiva institucional que permita atender lo cotidiano, entendido como lo normalizado, con todos los elementos de política pública educativa en clases presenciales, así como atender lo tiempos de crisis que implican la ruptura de una normalidad y que se puedan atender en línea o distancia.

Es decir, se han generado innovaciones ante esta ruptura con la normalidad que demuestran la necesidad de enfoques más eficientes y con miras de equidad que permitan reducir las 
diferencias en el aprendizaje de los alumnos de educación universitaria. Se considera que es fundamental aprender de estos resultados positivos y, de cierta manera, que sean incorporados a los procesos ordinarios. Para finalizar, es imperativo mencionar que este primer análisis de las políticas públicas educativas podría generar otros estudios desde la perspectiva posdecisional, con una mirada de evaluación, siempre y cuando regresen los estudiantes a las aulas de la UPV.

\section{Bibliografía}

Álvarez, M.; N. Gardyn; A. lardelevsky; G. Rebello (2020). Segregación educativa en tiempos de pandemia: Balance de las acciones iniciales durante el aislamiento social por el COVID-19 en Argentina. Revista Internacional de Educación para la Justicia Social, 9(3), 25-43. https://doi. org/10.15366/riejs2020.9.3.002

Aguilar, L. F. (1992). El estudio de las políticas públicas. México: Miguel Ángel Porrúa.

Aguilar, L. F. (2003). Problemas públicos y agenda de gobierno. México: Miguel Ángel Porrúa.

Baena, G. P. (2015). Planeación prospectiva estratégica. Teorías, metodologías y buenas prácticas en América Latina. México: Universidad Nacional Autónoma de México.

Banco Mundial (2020). COVID-19: Impacto en la educación y respuestas de política pública. Grupo Banco Mundial.

Bensa, A. (2015). Después de Lévi-Strauss. Por una antropología de escala humana. México: Fondo de Cultura Económica.

Bensa, A. (2016). El fin del exotisimo. Ensayos de antropología crítica. México: Colmich/Conaculta.

Expósito, C. D.; R. G. Marsollier (2020). Virtualidad y educación en tiempos de COVID-19. Un estudio empírico en Argentina. Educación y Humanismo, 22(39). https://doi.org/10.17081/eduhum.22.39.4214

Fuentes-Amaya, S. (2010). Estudio introductorio. Hacia una analítica de la política educativa. En Fuentes-Amaya, S.; O. P. Cruz-Pineda (eds.). Identidades y políticas educativas. México: UPN, 13-38.

Fuentes-Amaya, S. (2012). La construcción del objeto de estudio. Entre la demanda institucional y el oficio de investigar. En Jiménez, M. A. Investigación educativa. Huellas metodológicas. México: Juan Pablos Editor, 219-238.

Fuentes, N. F. (2018). Lo político y lo público en las políticas públicas. Una perspectiva político-discursiva. En Fuentes, N. F. (ed.). Políticas públicas y politicidad en educación. Configuraciones teóricas e investigativas. Xalapa: Universidad Pedagógica Veracruzana, 23-57.

Jacorzynski, W. (2004). Crepúsculo de los ídolos en la antropología social: más allá de Malinowski y los posmodernistas. México: CIESAS.

Diálo pos 
Lasswell, H. (1992). La orientación de las políticas públicas. En Aguilar, L. V. (ed.). El estudio de las políticas públicas. México: Miguel Ángel Porrúa, 79-103.

Majone, G. (2014). Evidencia, argumentación y persuación en la formulación de políticas. México: Fondo de Cultura Económica.

Mérida, Y.; L. A. Acuña (2020). COVID-19, pobreza y educación en Chiapas: Análisis a los programas educativos emergentes. Revista Internacional de Educación para la Justicia Social, 9(3), 61-82. https://doi.org/10.15366/riejs2020.9.3.004

Miklos, R. T.; M. E. Tello (2015). Planeación prospectiva: una estrategia para el diseño del futuro. México: LIMUSA.

Parsons, W. (2017). Políticas públicas: una introducción a la teoría y la práctica del análisis de políticas públicas. México: FLACSO.

Remedí, E. (2008). Sujetos, culturas, procesos en instituciones universitarias. IV Congreso Internacional de Análisis Organizacional. México: Red Mexicana de Investigadores en Estudios Organizacionales (REMINEO).

Restrepo, E. (2016). Etnografía: alcances, técnicas y éticas. Bogotá: Envión Editores.

Ruiz-Bolívar, C. (2008). El enfoque multimétodo en la investigación social y educativa: una mirada desde el paradigma de la complejidad. Revista de Filosofía y Socio Política de la Educación. 13-28.

Secretaría de Educación Pública (2020). Estrategia Aprende en Casa. Ciclo 2019-2020. https:// aprendeencasa.sep.gob.mx/site/index

Secretaría de Educación de Veracruz (2020). Veracruz educando a distancia. https://www.sev. gob.mx/educando-a-distancia/

Velasco, J. M.; P. Peredo; F. Fuentes; G. González; M. López (2016). Horizonte educativo. Hacia una pedagogía de la autoorganización. México: Universidad Pedagógica Veracruzana.

Villarreal, E. C. (2010). Políticas públicas. En Villareal, E. C.; V. H. Martínez (eds.). (Pre)textos para el análisis político. Disciplinas, reglas y procesos. México: FLACSO.

Viqueira, J. P. (2016). Después de Lévi-Strauss. Nexos, (1). México. Nexos. 